\title{
Review
}

Yuchi Jia*

\section{The progress in study of fosfomycin}

DOI: $10.1515 / \mathrm{ii}-2017-0162$

Received December 15, 2017; accepted January 12, 2018; published online April 10, 2018

\begin{abstract}
In recent years, heavy drug resistance in bacteria has become increasingly serious, and the progress of research and development of new antibiotics are slow. Therefore, seeking an alternative from the safe and effective "old medicine" is a strategy for clinical action against infection. Among them, fosfomycin is extremely relevant. Based on the related literature at home and abroad, this review briefly introduces fosfomycin in the context of progress in synthetic methods, pharmacokinetic and pharmacodynamic characteristics, and antibacterial activities, to provide references for clinical rational use.
\end{abstract}

Keywords: fosfomycin, synthesis, pharmacokinetics, antibacterial activity

\section{Introduction}

Fosfomycin is a phosphoric acid derivative extracted from several Streptomyces species in Spain soil in 1969 by Hendlin et al. [1]. Its molecular formula was determined as (-)-(1R, 2S)-1,2-epoxy propyl phosphonic acid by Christensen et al. [1]. Fosfomycin has a unique chemical structure; its molecular weight is very small, it has no antigenicity and does not show cross-resistance with other antibacterial drugs. Fosfomycin is a homologous variant of phosphoenolpyruvate. It can covalently bind to two phosphates of uridine diphosphate-Nacetylglucosamine transferase (MurA) and inhibit MurA activity irreversibly, thus blocking the first step of bacterial cytoderm synthesis effectively and then destroying the bacteria [2]. Fosfomycin is a rapid bactericide for the bacterial breeding season; it has excellent antibacterial activity against Gram-positive bacteria (such as Staphylococcus aureus and Enterococcus) and Gram-negative bacteria (such as Enterobacteriaceae, Haemophilus, Pseudomonas aeruginosa, and so on). In addition, fosfomycin also has good antibacterial effects on methicillin-resistant Staphylococcus aureus (MRSA), vancomycin-resistant Enterococcus (VRE), and extendedspectrum beta-lactamases-producing (ESBL) Escherichia coli [2-4]. In recent years, due to the increasing number of high-resistance bacteria, enzyme-producing strains, and multidrug-resistant strains, the known antibacterial agents need to be urgently reassessed. So, fosfomycin is considered an alternative treatment for some special infections. In the early years, fosfomycin was used for intravenous injection in patients with multiple infections (including meningitis).Now, in some European countries, fosfomycin is approved for soft tissue infection and sepsis because of its long serum half-life, good tissue distribution, and high concentration in urine. In the United States, the Food and Drug Administration (FDA) approved the treatment of fosfomycin is suitable for patients with simple bladder arthritis; in Turkey, fosfomycin oral preparation has been authorized for the treatment of urinary tract infections [5-7]. Some studies also suggest that high-dose intravenous injection of fosfomycin can achieve enough concentration in cerebrospinal fluid (CSF) and bone joints to treat osteoarthritis, pneumonia, and bacteremia, which are caused by carbapenem-resistant and multidrugresistant Enterobacteriaceae [8-11]. Currently, fosfomycin has been synthesized by artificial synthesis and has been widely applied in overseas countries. It is highly valued by numerous medical professionals and has advantages that other antibiotics cannot match. In China, fosfomycin has also been included in the national essential drugs list. The common forms of marketed/sold drugs are oral tablets of fosfomycin calcium salt, phosphonomycin amino butyl alcohol three salt granules, and fosfomycin two sodium salt for injection.

Department of Infection Research Institute, The Second Hospital of Tianjin Medical University, Tianjin 300211, People’s Republic of China

*Correspondence: Yuchi Jia, E-mail: 981966715@qq.com 
Although the clinical application of phosphomycin has increased in recent years, there are few clinical reports in China. According to the related literatures at home and abroad, this review briefly introduces fosfomycin in the following three aspects: progress in synthetic methods, pharmacokinetic and pharmacodynamic characteristics, and antibacterial activities, to provide references for clinical rational use.

\section{The synthesis of fosfomycin}

Fosfomycin was first successfully synthesized in China in 1972, and it was applied to the clinic in the 1980s. Due to the poor stability of fosfomycin, it must exist in the form of salt. The common forms are (-)-cis-1,2glycidyl phosphate $(+)$ phenylethylamine (Left turn right ammonium salt), fosfomycin sodium, calcium phosphonomycin, phosphonomycin ammonia butyl alcohol three, and so on. Because dextrofosfomycin is invalid, fosfomycin usually refers to fosfomycin. Fosfomycin phenylethylamine salt is always used for the synthesis of other phosphorus phosphonomycin medications, fosfomycin sodium is used for injection, and fosfomycin calcium phosphonomycin is used for oral administration. Fosfomycin trometamol overcomes some disadvantages of the metal salt and is more gentle and effective. The main synthetic methods of fosfomycin are biosynthesis and chemical synthesis.

\subsection{Biosynthesis method}

Specific biosynthetic enzymes or strains can be prepared by biosynthesis methods such as isolation of related gene, cloning, and enzyme catalysis. After isolation of genetic information from primary Streptomyces, some specific genes are inactivated and cloned into Streptomyces Pallas to successfully synthesize phosphomycin; however, the yield is too low, and the screening and synthesis of biological enzymes and strains are cumbersome and costly [12].

\subsection{Chemical synthesis method}

Fosfomycin is synthesized from propynol and phosphorus trichloride by substitution, hydrolysis, and rearrangement to prepare allene phosphate; hydrogenation then leads to cis-1-propenylphosphonic acid (cPPA); the ring body racemic oxidation of fosfomycin with hydrogen peroxide takes place and then $(+)$-alpha phenylethylamine split yields fosfomycin phenylethylamine salt [13]. This is the classic method for the preparation of L-fosfomycin phenylethylamine salt, but the completion time is long, and there are too many steps and too many by-products of cPPA catalytic hydrogenation; Moreover, unstable control, reaction stereoselectivity, high solvent consumption, and other issues still exist.

Chemical synthesis has many advantages, such as ease of obtaining raw materials, easy operation, mild reaction conditions, and easy disposal of waste. However, there are still problems such as poor stereoselectivity, low yield, and environmental pollution. Other methods do not meet the industrial requirements in terms of activity, selectivity, and operability. The highly efficient asymmetric catalysis of fosfomycin is the key to solving the problem of industrial synthesis of fosfomycin at home and abroad. It is also the focus of future research on fosfomycin synthesis.

\section{Advances in pharmacokinetics (PK) and pharmacodynamics (PD)}

The molecule of fosfomycin contains a propylene epoxy group, which is simple and unique in chemical structure and which makes it different from other antibiotics. Many clinical research reports are about the oral preparations (fosfomycin trometamol, fosfomycin calcium) and injection preparation (phosphomycin 
disodium salt). The oral bioavailability of fosfomycin trometamol is $34 \%-58 \%$, is widely distributed in tissue and body fluid after being absorbed, the apparent distribution volume is reported to be different (40-136 L) [14]. The oral gastrointestinal absorption of fosfomycin calcium in normal persons is $30 \%-40 \%$. After oral administration of $0.5 \mathrm{~g}, 1 \mathrm{~g}$, and $2 \mathrm{~g}$ fosfomycin calcium, the concentration of blood drug reached its peak at 2-4 hours. There are reports of patients and healthy volunteers after intravenous injection of fosfomycin: drugs can penetrate into the skin and muscle tissue, then into the lung; bone tissue and CSF can also achieve high concentration, but the concentration of drugs in the abdominal cavity is low. The highest concentration of drug in the blood can reach $606 \mathrm{mg} / \mathrm{L}$ [15]. The drug concentration decreases rapidly, and the concentration is reduced to half of the peak concentration about 1 hour later; however, the urine concentration is higher than the blood concentration. Fosfomycin is widely distributed in tissues: the highest concentration is in renal tissue, bladder wall, and urine. Secondly, the concentration is high in the heart, lung, liver, and other organs, and there is a certain concentration in the fetal circulation, bile, and milk. Fosfomycin can penetrate the blood-brain barrier and can enter the hydrothorax, ascites, lymph, bronchial secretions, and aqueous humor of the eye.

After fosfomycin enters the body, it is not degraded; it is excreted in the urine in the original form, and there is almost no excretion and reabsorption from renal tubules. About $85 \%-95 \%$ of the drug can be expelled after 24 hours. The concentration in blood and the time of elimination are closely related to the function of the kidney. We should pay more attention to the changes in the PK of antibiotics in special patients, such as newborns, elderly patients, patients with liver and kidney dysfunction, and so on. Due to the large volume and small glomerular filtration rate of newborns, the half-life of fosfomycin in neonatal patients is significantly prolonged, and it shows time-dependent antibacterial activity in neonates and children [16]. Compared with young adults, the serum levels of the drug in the elderly are significantly increased after oral administration of fosfomycin, and the clearance is significantly slowed down [17]. For critically ill patients, because of many physiological and pathological conditions, such as creatinine clearance rate change, the clinical dosage of fosfomycin can be doubled or the dose reduced, to avoid inadequate dose or poisoning [18]. Fosfomycin can be removed using artificial kidney, so the dose should not be adjusted for patients with continuous veno-venous hemofiltration (CVVH) [19]. It is also reported that the automated peritoneal dialysis patients without peritonitis showed good systemic distribution after intraperitoneal administration of fosfomycin, but the permeability of peritoneal fluid after intravenous administration is very low [20]. This cited study can provide reference for the clinical application of phosphomycin for special patients.

The concept of clinical antibiotic application has been widely recognized in recent years through the theory of PK/PD. According to the PK/PD characteristics of different antimicrobial agents, designing reasonable antimicrobial regimens is of great significance for improving efficacy, reducing drug resistance, and reducing adverse reactions [21]. However, there are few reports about whether fosfomycin is a timedependent or concentration-dependent antibiotic. Existing studies have shown that dependence is different due to different microorganisms. For example, fosfomycin shows the disinfection characteristics of time dependence of Staphylococcus aureus and Pseudomonas aeruginosa [22], but in the case of differentiation from urinary tract infection caused by fecal enterococcus and Escherichia coli, fosfomycin shows a concentration-dependent bactericidal effect [23,24]. At the same time, attention should be paid to the increase in the concentration of fosfomycin, and the effect of the antibiotics on E. coli (postantibiotic effect [PAE]) should also be extended, indicating that the dosage should be increased appropriately and the drug interval should be extended without affecting the PD.

\section{The antibacterial activity of fosfomycin}

Fosfomycin has a broad-spectrum disinfection role in the early stages of the synthesis of bacterial cell walls, with fast sterilization in the breeding season. Fosfomycin is resistant to Gram-positive coccus (such as Staphylococcus, Streptococcus, Pneumococcus); Gram-negative bacilli (such as Escherichia coli, Saracia, Shigella, 
Yersinia, most Pseudomonas aeruginosa, Klebsiella pneumoniae, and Vibrio). It also has some antibacterial effects on Proteus, bacilli, and some anaerobes but is weaker than penicillin and cephalosporin. Fosfomycin also acts against MRSA, ultra-broad-spectrum beta lactamase-producing (or ESBL) E. coli and Klebsiella pneumonia, and classes of VRE. However, Acinetobacter baumannii, fragile bacilli, and anaerobic Grampositive coccus are the naturally resistant to fosfomycin [25]. Bacteria usually do not show cross-resistance between fosfomycin and other antibiotics [26], and because it can destroy the outer structure of the bacteria or change the drug's access to the bacteria, the drug can be easily enriched in the bacteria and thus have a good synergistic effect with many antibiotics.

Fosfomycin has good synergistic effect with other antibiotics (beta lactams, aminoglycosides, quinolones, and macrolides) in the treatment of non-urinary tract infection and can reduce the minimum inhibitory concentration (MIC) value of drug-resistant bacteria to the combined drugs [27]. At the same time, fosfomycin can reduce the toxicity of some antibacterial drugs on the body, such as glycopeptides and aminoglycosides, combined with reduced renal toxicity; combined with aminoglycosides, polymyxin can reduce the occurrence of ototoxicity [15] because fosfomycin can destroy bacterial cell wall and biofilm, then create conditions for the accumulation of other antimicrobial agents.

In recent years, heavy drug resistance in bacteria has become increasingly serious, and the progress of research and development of new antibiotics are slow; so seeking alternatives from the safe and effective "old medicine" is a strategy for clinical action against infection. Fosfomycin still has high antibacterial activity against Gram-positive and Gram-negative bacteria, including some drug-resistant bacteria, such as MRSA, ESBL, and VRE, and has been well tolerated in different populations. Therefore, the old drug fosfomycin has a broad application prospect in the treatment of difficult and severe infection.

Conflict of Interest: The author states no conflicts of interest.

\section{References}

[1] Itoh N., Kusaka M., Hirota T., Nomura A., Microbial production of antibiotic fosfomycin by a stereoselective epoxidation and its formation mechanism, Appl. Microbiol. Biotechnol., 1995, 43, 394-401.

[2] Raz R., Fosfomycin: an old - new antibiotic, Clin. Microbiol. Infect., 2012, 18(1), 4-7.

[3] Karageorgopoulos D.E., Wang R., Yu X.H., Falagas M.E., Fosfomycin: evaluation of the published evidence on the emergence of antimicrobial resistance in Gram-negative pathogens, J. Antimicrob. Chemother., 2012, 67, 255-268.

[4] Wu N., Chen B., et al., Antiseptic effect of fosfomycin on Escherichia coli producing super broad-spectrum beta lactamase, Chin J. Infect. Chemother, 2013, 13(2), 140-142.

[5] Demir T., Buyukgucluc T., Fosfomycin: In vitro efficacy against multidrug-resistant isolates beyond urinary isolates, J. Global Antimicrob. Resistance, 2017, 8, 164-168.

[6] Demir T., Buyukguclu T., Evaluation of the in vitro activity of fosfomycin tromethamine against Gram-negative bacterial isolates recovered from community- and hospital-acquired urinary tract infections in Turkey, Int. J. Infect. Dis., 2013, 17, 966-970.

[7] Popovic M., Steinort D., Pillai S., Joukhadar C., Fosfomycin: an old, new friend? Eur. J. Clin. Microbiol. Infect. Dis., 2010, 29, 127-142.

[8] Falagas M.E., Maraki S., Karageorgepoulos D.E., Kastoris A.C., Mavromanalakis E., Samonis G., Antimicrobial susceptibility of multidrug-resistant (MDR) and extensively drug-resistant (XDR) Enterobacteriaceae isolates to fosfomycin, Int. J. Antimicrob. Agents, 2010, 35, 240-243.

[9] Michalopoulos A.S., Livaditis I.G., Gougoutas V., The revival of fosfomycin, Int. J. Infect. Dis., 2011, 15, 732-739.

[10] Samonis G., Maraki S., Karageorgopoulos D.E., Vouloumanou E.K., Falagas M.E., Synergy of fosfomycin with carbapenems, colistin, netilmicin, and tigecycline against multidrug-resistant Klebsiella pneumoniae, Escherichia coli, and Pseudomonas aeruginosa clinical isolates, Eur. J. Clin. Microbiol. Infect. Dis., 2012, 31, 695-701.

[11] del Río A., Gasch O., Moreno A., Peña C., Cuquet J., Soy D., et al., FOSIMI Investigators: Efficacy and safety of fosfomycin plus imipenem as rescue therapy for complicated bacteremia and endocarditis due to methicillin resistant Staphylococcus aureus: a multicenter clinical trial, Clin. Infect. Dis., 2014, 59, 1105-1112.

[12] Hendin D., Stapley E.O., Jackson M., Phosphomycin, Science, 1969, 166, 122-123.

[13] Glamkowski E, Rosas C, Sletzinger M, et al. Process for the preparation of cis-1- propenylphosphonic acid. US3733356A, 1973-5-15. 
[14] Rizek C., Ferraz J.R., van der Heijden I.M., In vitro activity of potential old and new drugs against multidrug resistant gram-negatives, J. Infect. Chemother., 2015, 21(2), 114-117.

[15] Roussos N., Karageorgopoulos D.E., Samonis G., Falagas M.E., Clinical significance of the pharmacokinetic and pharmacodynamic characteristics of fosfomycin for the treatment of patients with systemic infections, Int. J. Antimicrob. Agents, 2009, 34(6), 506-515.

[16] Traunmuller F., Popovic M., Konz K.H., Vavken P., Leithner A., Joukhadar C., A reappraisal of current dosing strategies for intravenous fosfomycin in children and neonates, Clin. Pharmacokinet., 2011, 50(8), 493-503.

[17] Borsa F., Leroy A., Fillastre J.P., Comparative pharmacokinetics of tromethamine fosfomycin and calcium fosfomycin in young and elderly adults, Antimicrob. Agents Chemother, 1988, 32(6), 938-941.

[18] Parker S., Lipman J., Koulenti D., Dimopoulos G., Roberts J.A., What is the relevance of fosfomycin pharmacokinetics in the treatment of serious infections in critically ill patients? A systematic review, Int. J. Antimicrob. Agents, 2013, 42(4), 289-293.

[19] Gattringer R., Meyer B., Heinz G., Guttmann C., Zeitlinger M., Joukhadar C., Single-dose pharmacokinetics of fosfomycin during continuous venovenous haemofiltration, J. Antimicrob. Chemother, 2006, 58(2), 367-371.

[20] Tobudic S., Matzneller P., Stoiser B., Wenisch J.M., Zeitlinger M., Vychytil A., Pharmacokinetics of intraperitoneal and intravenous fosfomycin in automated peritoneal dialysis patients without peritonitis, Antimicrob. Agents Chemother., 2012, 56(7), 3992-3995.

[21] Chen B., Optimization of antimicrobial therapy under the guidance of PK/PD theory, Chin. J. Clin. Infect. Dis, 2009, 2(2), 69-70.

[22] MacLeod D.L., Velayudhan J., Kenney T.F., Therrien J.H., Sutherland J.L., Barker L.M., et al., Fosfomycin enhances the active transport of tobramycin in Pseudomonas aeruginosa, Antimicrob. Agents Chemother, 2012, 56(3), 1529-1538.

[23] Descourouez J.L., Jorgenson M.R., Wergin J.E., Fosfomycin synergy in vitro with amoxicillin, Antimicrob. Agents Chemother., 2013, 57(3), 1518-1520.

[24] Mazzei T., Cassetta M.I., Fallani S., Pharmacokinetic and pharmacodynamic aspects of antimicrobial agents for the treatment of uncomplicated urinarytract infections, Int. J. Antimicrob. Agents, 2006, 28(Suppl. 1), S35-S41.

[25] Quan J., Lai B., Research status of clinical application of fosfomycin, Chin. J. Clin. Pharmacol., 2015, 31(20), 2067-2069.

[26] Expert Committee on Rational use of Drugs by the Ministry of Health. Clinical medication guide for Chinese pharmacists, Chongqing Press, Chongqing, 2009, 169-170.

[27] Lian J., Tang Y., Synergistic effect of combined use of sodium fosfomycin sodium and other antibiotics and its clinical application, Foreign Med. Antibiot. Vol., 2003, 24(1), 44-45. 